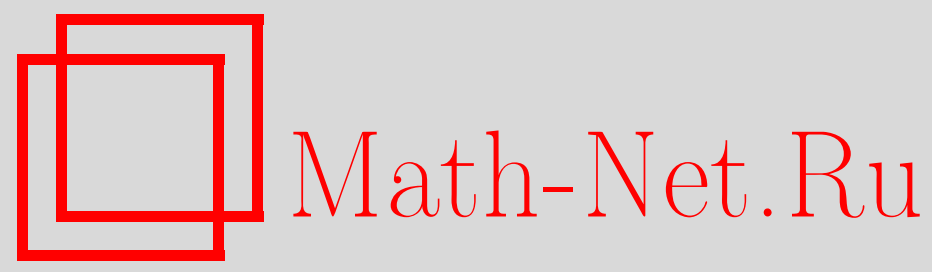

Г. Г. Ильюта, Высшие порядки Брюа и соотношения в группе кос, Матем. заметки, 1999, том 66, выпуск 6, 840-848

DOI: https://doi.org/10.4213/mzm1228

Использование Общероссийского математического портала Math-Net.Ru подразумевает, что вы прочитали и согласны с пользовательским соглашением http://www . mathnet.ru/rus/agreement

Параметры загрузки:

IP: 3.93.64.190

26 апреля 2023 г., 15:52:10

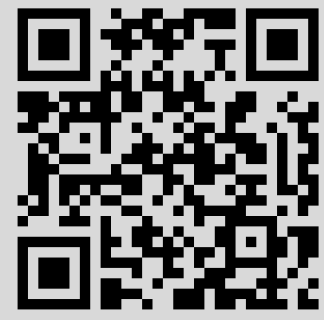




\section{ВЫСШИЕ ПОРЯДКИ БРЮА И СООТНОШЕНИЯ В ГРУППЕ КОС}

\section{Г.Г. Ильюта}

В статье доказаны соотношения в группе кос, которые обобщают соотношение вида $a b a=b a b$. Они получаются с помощью обобщения на высшие порядки Брюа известного соответствия между разложениями элемента максимальной длины в группе Вейля $A_{n-1}$ и специального вида линейными порядками на системе положительных корней $A_{n-1}$.

Библиографоия: 10 названий.

Наиболее естественным высшим аналогом отражения в конечной группе Кокстера, по-видимому, следует считать элемент наибольшей длины. Например, в отличие от другого претендента - элемента Кокстера - он имеет порядок 2 и не зависит от нумерации вершин соответствующей диаграммы Кокстера-Дьнкина. Элемент Кокстера имеет другое преимущество - он определен и для бесконечных груп, порожденных отражениями, и, например при изучении распадений особенностей [1], именно элемент Кокстера (оператор классической монодромии) появляется как обобщенное отражение. В этой статье доказаны соотношения в симметрической группе (т.е. в групше Кокстера $A_{n-1}$ ) и в групе кос, которые обобщают соотношение $a b a=b a b$ и в которых роль обобщенных отражений играют элементы наибольшей длины в подгруппах симметрической группы (в групу кос они поднимаются как обобщения фундаментального элемента Гарсайда).

Доказанные соотношения тесно связаны с возникаюшими в теории высших порядков Брюа [2] специального вида линейньпи порядками (будем называть их допустимыми) на множестве $k$-подмножеств в $\bar{n}=\{1, \ldots, n\}$. Сопоставим $k$-подмножеству $\left\{i_{1}, \ldots, i_{k}\right\} \subset \bar{n}, i_{1}<\cdots<i_{k}$, элемент наибольшей длины в группе перестановок на этом подмножестве, т.е. перестановку $\left(i_{1}, \ldots, i_{k}\right) \rightarrow\left(i_{k}, \ldots, i_{1}\right)$. В группу кос эта перестановка поднимается как обобщение фундаментального элемента Гарсайда (определение в следующем абзаце). Теперь для фиксированного $k$ можно рассматривать произведения (например, в допустимых порядках) по всем $k$-подмножествам сопоставленных этим $k$-подмножествам элементов. Для $k=n-1$ имеется лишь два допустимых порядка. Основной результат статьи состоит в доказательстве совпадения соответствуюших этим двум порядкам произведений в симметрической групе и для четного $n$ в группе кос. Отсюда следует, что для любого $k \leqslant n$ в симметрической группе совпадают произведения, отвечающие допустимым порядкам, которые отличаются лишш инверсиями (определение инверсии см. в п. 1). Это следствие обобщает (частично, по- 
скольку для $2<k<n-1$ инверсии не транзитивны на допустимых порядках) известньй для $k=2$ факт, а именно: транспозиции в симметрической группе взаимно однозначно соответствуют 2 -подмножествам в $\bar{n}$ и произведение всех транспозиций в любом допустимом порядке совпадает с элементом наибольшей длины, т.е. с перестановкой $(1, \ldots, n) \rightarrow(n, \ldots, 1)[3],[4]$.

Для точной формулировки результата введем обозначения. В групше кос

$$
B_{n}=\left\langle b_{1}, \ldots, b_{n-1}\left|b_{i} b_{i+1} b_{i}=b_{i+1} b_{i} b_{i+1}, b_{j} b_{m}=b_{m} b_{j},\right| j-m \mid>1\right\rangle
$$

рассмотрим элементы

$$
h(i, j)=\prod_{m=i}^{j} b_{m}, \quad b(i, j)=h(i, j) b_{j}^{-1} h(j, i)
$$

(знак П используется в статье для обозначения произведений с некоммутирующими сомножителями и предполагается, что перемножение производится в порядке изменения индекса от нижнего до верхнего, причем индекс может как возрастать, так и убывать). Пусть для $I=\left\{i_{1}, \ldots, i_{k}\right\} \subset \bar{n}, i_{1}<\cdots<i_{k}$,

$$
e_{I}=\prod_{j=1}^{[k / 2]} b\left(i_{j}, i_{k+1-j}-1\right), \quad e_{i}=e_{\bar{n} \backslash\{i\}} .
$$

Согласно лемме 4 в п. $1 e_{\bar{n}}$ совпадает с фундаментальным элементом Гарсайда. В статье для четного $n$ доказано следуюшее соотношение в $B_{n}$ :

$$
\prod_{i=1}^{n} e_{i}=\prod_{i=n}^{1} e_{i}
$$

В групе перестановок такое соотношение (с заменой $b_{i}$ на транспозицию $(i, i+1)$ доказано для любого $n$. Например, для $n=4$ имеем

$$
b_{1} b_{2} b_{1}\left(b_{1} b_{2} b_{3} b_{2} b_{1}\right)^{2} b_{2} b_{3} b_{2}=b_{2} b_{3} b_{2}\left(b_{1} b_{2} b_{3} b_{2} b_{1}\right)^{2} b_{1} b_{2} b_{1} \text {. }
$$

Высшие порядки Брюа были введены Ю. А. Маниным и В. В. Шехтманом при изучении многомерного уравнения Янга-Бакстера. В теории уравнений Янга-Бакстера высшие соотношения в группе кос отвечают построению решений многомерных уравнений Янга-Бакстера из решений двумерного уравнения [5] (поскольку высшие соотношения в групе кос являются, конечно же, следствиями определяющих соотношений). В серии соотношений в группе кос, анонсированной в [5], приведенному вьше соотношению для $n=4$ по размерности отвечает соотношение

$$
b_{1} b_{2} b_{1} b_{2} b_{3} b_{2} b_{1} b_{2} b_{1} b_{2} b_{3} b_{2}=b_{2} b_{3} b_{2} b_{1} b_{2} b_{1} b_{2} b_{3} b_{2} b_{1} b_{2} b_{1} \text {. }
$$

В п. 4 мы выразим через фундаментальньй элемент Гарсайда левую и правую части соотношений из [5].

С каждым допустимьм порядком на транспозициях из симметрической групшы связаны две таблищы Юнга [3], [4]. Очевидное обобщение для одной из этих таблищ для $k>2$ приводится в [6] (используются многомерные таблицы Юнга). В п. 5, исходя из рассмотрения элемента наибольшей длины как обобщения транспозиции, мы сопоставим для четного $k$ каждому допустимому порядку обычную двумерную таблицу Юнга. Возможно, такой подход окажется более перспективным для ответа на вопрос Манина и Шехтмана [2] о перенесении на допустимые порядки на $k$-множествах в $\bar{n}$ результатов [4]. 
1. Обозначения и вспомогательные формулы. Пусть $S_{n}$ - группа перестановок на $\bar{n}, \pi: B_{n} \rightarrow S_{n}: b_{i} \mapsto(i, i+1)$ - естественньй эпиморфизм, $R_{n}$ - автоморфизм групшы $B_{n}$, действующий на образующих следующим образом: $R_{n}\left(b_{i}\right)=b_{n-i}[7]$, $\omega_{n}:(1, \ldots, n) \rightarrow(n, \ldots, 1)$ - элемент наибольшей длины в $S_{n}$ относительно множества порождающих $(i, i+1), i=1, \ldots, n-1$,

$$
\Delta_{n}=\prod_{i=n-1}^{1} h(1, i)
$$

- фундаментальньй элемент Гарсайда в $B_{n}[7]$. Известно, что $\pi\left(\Delta_{n}\right)=\omega_{n}[8]$.

ЛЕмма 1. $b(i, j)=b(j, i)$.

ДокАЗАТЕЛЬСТво. Для $i=j$ равенство вьполняется; для $|i-j|=1$ оно совпадает с одним из определяющих соотношений в группе кос; осталось доказать его для $|i-j|>1$. Доказьваем по индукции:

$$
\begin{aligned}
b(i, j) & =h(i, j-2) b_{j-1} b_{j} b_{j-1} h(j-2, i)=h(i, j-2) b_{j} b_{j-1} b_{j} h(j-2, i) \\
& =b_{j} h(i, j-2) b_{j-1} h(j-2, i) b_{j}=b_{j} b(i, j-1) b_{j}=b_{j} b(j-1, i) b_{j}=b(j, i) .
\end{aligned}
$$

ЛЕмма 2 [7]. Справедливы следующие соотношения:
а) $b_{k} h(i, j)= \begin{cases}h(i, j) b_{k-1}, & \text { если } i<k \leqslant j, \\ h(i, j) b_{k+1}, & \text { если } i \geqslant k>j\end{cases}$
б) $R_{n}\left(\Delta_{n}\right)=\Delta_{n}$
в) $b \Delta_{n}^{2 m-1}=\Delta_{n}^{2 m-1} R_{n}(b), b \Delta_{n}^{2 m}=\Delta_{n}^{2 m} b, b \in B_{n}, m \in \mathbb{Z}$.

Следующая лемма доказьвается многократным применением соотношения а) леммы 2 .

Лемма 3. Если $i<k \leqslant m<j$, mo $b(i, j) b(k, m)=b(k, m) b(i, j)$.

ЛЕмма 4. $\Delta_{n}=\prod_{i=1}^{[n / 2]} b(i, n-i)$.

ДокАЗАТЕЛЬСТВо. Поскольку $b(i, j)$ является поднятием транспозиции, т.е.

$$
\pi(b(i, j))=\left\{\begin{array}{l}
(i, j+1), \text { если } i \leqslant j, \\
(i+1, j), \text { если } i \geqslant j,
\end{array}\right.
$$

то $\pi$ переводит правую часть доказьваемого равенства в $\omega_{0}$. В разложении правой части по образующим столько же элементов, сколько и в разложении $\Delta_{n}$ из определения. Лемма вытекает из этих двух замечаний согласно предложению 5.7 (ii) из [8]. 
ЗАмЕчаниЕ. Согласно лемме 3 равенство леммы 4 представляет $\Delta_{n}$ в виде произведения коммутирующих элементов. В теории конечных груп Кокстера этот факт соответствует известному представлению элемента наибольшей длины в группе в виде произведения коммутирующих инволюций. Коммутирование сомножителей в равенстве леммы 4 является ключевым фактом в доказательстве основных результатов статьи.

Определим допустимые порядки на множестве $C(n, k) k$-подмножеств в $\bar{n}$. Полное определение высших порядков Брюа нам не понадобится, его можно найти в [2].

Для $e \in C(n, k+1)$ назовем пакетом в $C(n, k)$ множество $\{d: d \in C(n, k), d \subset e\}$. Например, единственный пакет в $C(n, n-1)$ совпадает с $C(n, n-1)$. На $C(n, k)$ имеется естественньй линейный порядок - лексикографический. Произольньй порядок на $C(n, k)$ назовем допустимылм, если на каждом пакете он индуцирует либо лексикографический порядок, либо обратный лексикографическому. В частности, любой линейный порядок на $C(n, 1)$ является допустимым и множество линейных порядков на $C(n, 1)$ совпадает с множеством перестановок $\bar{n}$.

Скажем, что два допустимых порядка связаны инверсией, если они отличаются порядком на единственном пакете, элементы которого расположены в обоих допустимых порядках последовательно (между ними нет элементов, не принадлежащих этому пакету).

2. В этом пункте доказан основной результат статьи.

Tеорема 1. Для четного $n$

$$
\prod_{i=1}^{n} e_{i}=\prod_{i=n}^{1} e_{i}
$$

Доказательство вытекает из следующих двух лемм. Введем обозначения

$$
E(i, j)=\prod_{k=j}^{i} e_{k}, \quad \Delta(i, j)=\prod_{k=j-1}^{i} h(i, k)
$$

В частности, $\Delta(1, n)=\Delta_{n}$.

Лемма 5. Имеет место равенство

$$
E(1, n)=\Delta_{n} h(2, n-1) E(2, n-1)(b(1, n-1))^{n-4} h(1, n-2) \Delta_{n}
$$

ДоКАЗАТЕЛЬСТво. По определению в $e_{i}, i=2, \ldots, n-1$, входит множитель $b(1, n-1)$, и согласно лемме 3 он коммутирует со всеми другими множителями в $e_{i}, i=2, \ldots, n-1$. Поэтому все множители $b(1, n-1)$ можно вьнести из произведения $e_{n-1} \cdots e_{2}$, и то, что останется от этого произведения, также по определению совпадает с $E(2, n-1)$ :

$$
E(1, n)=e_{n} b(1, n-1) E(2, n-1)(b(1, n-1))^{n-4} b(1, n-1) e_{1}
$$


Согласно лемме 4

$$
e_{n}=\Delta_{n-1}=\prod_{i=n-2}^{1} h(1, i)
$$

По лемме 3 из [7]

$$
\Delta_{n-1}=\prod_{i=1}^{n-2} h(1, i)
$$

Поэтому по лемме 1

$$
e_{n} b(1, n-1)=e_{n} b(n-1,1)=\left(\prod_{i=1}^{n-2} h(i, 1)\right) h(n-1,1) h(2, n-1)=\Delta_{n} h(2, n-1)
$$

По лемме $1 R_{n}\left(e_{1}\right)=e_{n}$. Поэтому

$$
\begin{aligned}
b(1, n-1) e_{1} & =R_{n}\left(b(n-1,1) \Delta_{n-1}\right)=R_{n}\left(h(n-1,2) h(1, n-1) \prod_{i=n-2}^{1} h(1, i)\right) \\
& =R_{n}\left(h(n-1,2) \Delta_{n}\right)=h(1, n-2) \Delta_{n} .
\end{aligned}
$$

ЛЕмма 6. Для четного $n$

$$
\prod_{i=n}^{1} e_{i}=\prod_{i=1}^{n / 2} b_{2 i-1}^{-1} \Delta_{n}^{n-1}
$$

ДокАЗАТЕльСТво. Доказьваем по индукции, используя лемму 5:

$$
\begin{aligned}
\prod_{i=n}^{1} e_{i} & =E(1, n)=\Delta_{n} h(2, n-1) E(2, n-1)(b(1, n-1))^{n-4} h(1, n-2) \Delta_{n} \\
& =\Delta_{n} h(2, n-1) \prod_{i=1}^{(n-2) / 2} b_{2 i}^{-1}(\Delta(2, n-1))^{n-3}(b(1, n-1))^{n-4} h(1, n-2) \Delta_{n}
\end{aligned}
$$

По лемме 4

$$
\Delta(2, n-1)=\prod_{i=2}^{n / 2} b(i, n-i)
$$

Поэтому $\Delta(2, n-1) b(1, n-1)=\Delta_{n}$ и

$$
\prod_{i=n}^{1} e_{i}=\Delta_{n} h(2, n-1) \prod_{i=1}^{(n-2) / 2} b_{2 i}^{-1} \Delta_{n}^{n-3}(b(1, n-1))^{-1} h(1, n-2) \Delta_{n}
$$


Пользуясь соотношением в) леммы 2 , собираем вместе все $\Delta_{n}$ :

$$
\prod_{i=n}^{1} e_{i}=h(n-2,1) \prod_{i=1}^{(n-2) / 2} b_{n-2 i}^{-1}(b(n-1,1))^{-1} h(1, n-2) \Delta_{n}^{n-1} .
$$

Аналогично доказательству соотношения а) леммы 2 с использованием соотношений

$$
b_{i} b_{i+1} b_{i}^{-1}=b_{i+1}^{-1} b_{i} b_{i+1}
$$

получаем равенство $h(n-2,1) b_{i}^{-1}=b_{i-1}^{-1} h(n-2,1)$. Поэтому, используя лемму 1 , имеем

$$
\prod_{i=n}^{1} e_{i}=\prod_{i=1}^{(n-2) / 2} b_{n-2 i-1} h(n-2,1)(b(1, n-1))^{-1} h(1, n-2) \Delta_{n}^{n-1}=\prod_{i=1}^{n / 2} b_{2 i-1} \Delta_{n}^{n-1} .
$$

ДОКАЗАТЕЛЬСТво ТЕОРЕМЫ 1. По Лемме 1

$$
R_{n}\left(\prod_{i=n}^{1} e_{i}\right)=\prod_{i=1}^{n} e_{i}
$$

Поскольку все $b_{2 i-1}$ коммутируют между собой, согласно соотношению б) леммы 2

$$
R_{n}\left(\prod_{i=1}^{n / 2} b_{2 i-1} \Delta_{n}^{n-1}\right)=\prod_{i=1}^{n / 2} b_{n-2 i-1} \Delta_{n}^{n-1}=\prod_{i=1}^{n / 2} b_{2 i-1} \Delta_{n}^{n-1}
$$

Теорема доказана.

Приведем еще две формулы, которые делают более понятным различие между четным и нечетньм $n$ в теореме 1 . Доказательство этих формул легко получается по индукции с использованием рекуррентного соотношения из леммы 5 . Напомним, что $R_{n}^{2}=\mathrm{Id}$, и поэтому

$$
R_{n}^{i}= \begin{cases}\mathrm{Id} & \text { для четных } i, \\ R_{n} & \text { для нечетных } i .\end{cases}
$$

ПРЕДЛОЖЕНИЕ 1. а) ДЛя четныХ $n$

$$
\prod_{i=n}^{1} e_{i}=\prod_{i=1}^{n / 2} R_{n}^{i+1}(h(n-i-1, i)) \Delta_{n} \prod_{i=n / 2}^{1} R_{n}^{i+1}(h(i, n-i-1)) \Delta_{n}^{n-3} .
$$

б) Для нечетных $n$

$$
\prod_{i=n}^{1} e_{i}=\prod_{i=1}^{(n-1) / 2} R_{n}^{i+1}(h(n-i-1, i)) \prod_{i=(n-1) / 2}^{1} R_{n}^{i+1}(h(i, n-i-1)) \Delta_{n}^{n-2} .
$$




\section{3. Результаты о симметрической группе.}

Теорема 2. а) Имеет место равенство

$$
\pi\left(\prod_{i=n}^{1} e_{i}\right)=\pi\left(\prod_{i=1}^{n} e_{i}\right)
$$

б) Для четных $n$

$$
\pi\left(\prod_{i=n}^{1} e_{i}\right)=\prod_{i=1}^{n / 2}(2 i-1,2 i) \omega_{n}
$$

в) Для нечетных $n$

$$
\pi\left(\prod_{i=n}^{1} e_{i}\right)=\omega_{n}
$$

ДокАЗАтЕльство. Для четных $n$ теорема 2 следует из теоремы 1. Утверждение в) доказьвается аналогично лемме 6 по индукции. Оно также вытекает из утверждения б) предложения 1 , поскольку

$$
\pi\left(\Delta_{n}\right)=\omega_{n}, \quad \omega_{n}^{2}=\mathrm{Id}, \quad \pi(h(i, j) h(j, i))=\mathrm{Id} .
$$

Утверждение а) для нечетных $n$ следует из утверждения в) так же, как теорема 1 следует из леммы 6. Теорема доказана.

Сопоставим допустимому порядку $d \in A(n, k)$ перестановку

$$
E_{d}=\prod_{I \in C(n, k)} \pi\left(e_{I}\right) \in S_{n}
$$

(сомножители расположены в порядке $d$ ).

Из теоремы 2 вытекает

СлЕДСТВИЕ. Если допустимые порядки $d_{1}, d_{2} \in A(n, k)$ связаны инверсией, то $E_{d_{1}}=E_{d_{2}}$.

ЗАмечАниЕ. Тем же методом обобщения можно доказать теоремы 1 и 2 для более общего определения пакета. А именно, для $m \geqslant 1$ назовем $m$-nакетом множество

$$
\{\bar{n} \backslash\{1, \ldots, m\}, \bar{n} \backslash\{2, \ldots, m+1\}, \ldots, \bar{n} \backslash\{n-m+1, \ldots, n\}\} .
$$

В определении высших порядков Брюа и в теоремах 1, 2 использовались 1-пакеты.

4. В этом пункте вычисляются в терминах фундаментального элемента Гарсайда $\Delta_{n}$ левая и правая части анонсированных в [5] соотношений в групе кос. В наших обозначениях соотношения из [5] выглядят следующим образом:

$$
\Delta(1, n-1) \Delta(2, n) \Delta(1, n-1) \cdots=\Delta(2, n) \Delta(1, n-1) \Delta(2, n) \cdots
$$

(по $n$ сомножителей с каждой стороны). Например, для $n=3 \Delta(1,2)=b_{1}, \Delta(2,3)=b_{2}$, $b_{1} b_{2} b_{1}=b_{2} b_{1} b_{2}$; для $n=4 \Delta(1,3)=b_{1} b_{2} b_{1}, \Delta(2,4)=b_{2} b_{3} b_{2}$, и получается указанное во введении соотношение. 
ПРЕДЛОЖЕНИЕ 2. Левая и правая части соотношения (1) равны $\Delta_{n}^{n-2}$.

ДоКАЗАТЕЛЬСТво. По определению

$$
\Delta(1, n-1)=\Delta_{n-1}=(h(1, n-1))^{-1} \Delta_{n} .
$$

По лемме 1 и соотношению б) леммы 2

$$
\Delta(2, n)=R_{n}(\Delta(1, n-1))=(h(n-1,1))^{-1} \Delta_{n} .
$$

Согласно соотношению в) леммы 2

$$
\Delta_{n} h(1, n-1)=h(n-1,1) \Delta_{n} .
$$

Поэтому

$$
\begin{aligned}
\Delta(1, n-1) \Delta(2, n) \Delta(1, n-1) \cdots & =(h(1, n-1))^{-n} \Delta_{n}^{n}, \\
\Delta(2, n) \Delta(1, n-1) \Delta(2, n) \cdots & =(h(n-1,1))^{-n} \Delta_{n}^{n} .
\end{aligned}
$$

Согласно [9]

$$
(h(n-1,1))^{n}=(h(1, n-1))^{n}=\Delta_{n}^{2} .
$$

\section{5. Задачи.}

ЗАДАчА 1. Следующее определение обобщает на $A(n, 2 m)$ одно из двух отображений из $A(n, 2)$ в множество таблиц Юнга [3]. Задача состоит в том, чтобы определить обобщение второго отображения и описать их связи аналогично [3].

Пусть $m \leqslant n / 2$. Занумеруем в лексикографическом порядке элементы $C(n, m)$. Тогда любому $\left\{i_{1}, \ldots, i_{2 m}\right\} \in C(n, 2 m), i_{1}<\cdots<i_{2 m}$, отвечает пара чисел $i<j$, где $i$ является номером $\left\{i_{1}, \ldots, i_{m}\right\}$, а $j$ - номером $\left\{i_{m+1}, \ldots, i_{2 m}\right\}$. Тем самьм, любому линейному порядку на $C(n, 2 m)$ (и, в частности, любому элементу $A(n, 2 m))$ соответствует таблица Юнга, в которой на месте $i j$ стоит номер в линейном порядке элемента из $C(n, 2 m)$, соответствующего $i j$ (предполагается, что таблища Юнга состоит из тех клеток $i j$, которым соответствует элемент $C(n, 2 m))$.

ЗАДАчА 2. Найти определяющие соотношения для групп

$$
\left\langle e_{I}|| I \mid=k\right\rangle \subset B_{n}, \quad\left\langle\pi\left(e_{I}\right)|| I \mid=k\right\rangle \subset S_{n} .
$$

ЗАДА ЧА 3. В [9], [10] доказано следуюшее обобщение соотношения $a b a=b a b$ в группе кос $B_{n}$ (гипотеза Кокстера): если $P=b_{1} b_{3} \cdots, Q=b_{2} b_{4} \cdots$, то $P Q P \cdots=Q P Q \cdots$ (по $n$ сомножителей с каждой стороны). Существует ли аналог этого соотношения для групп из задачи 2 ? 


\section{СПИСОК ЦИТИРОВАННОЙ ЛИТЕРАТУРЫ}

[1] Арнольд В.И., Варченко А.Н., Гусейн-Заде С. М. Особенности дифференцируемых отображений. Т. 2. М.: Наука, 1984.

[2] Manin Y., Schechtman V. Arrengements of hyperplanes, higher braid groups and higher Bruhat orders // Adv. Stud. Pure Math. 1985. V. 44. P. 289-308.

[3] Edelman P., Greene C. Balanced tableaux // Adv. Math. 1987. V. 63. P. 42-99.

[4] Stanley R. On the number of reduced decomposition of elements of Coxeter groups // European J. Combin. 1984. V. 5. P. 359-372.

[5] Li Y., Hu Z. Solution of $n$-simplex equation from solutions of braid group representation // J. Phys. A. 1995. V. 28. P. L219-L223.

[6] Ильюта Г. Г. Геометрические реализации высших порядков Брюа и $M$-морсификации // Изв. РАН. Сер. матем. 1996. Т. 60. №6. С. 91-100.

[7] Гарсайд Ф. Группа кос и другие группы // Математика (сб. переводов). 1970. Т. 14. № 4. C. 116-132.

[8] Брискорн Э., Сайто К. Группы Артина и группы Кокстера // Математика (сб. переводов). 1974. Т. 18. №3. С. 56-79.

[9] Enomoto H., Mizutani H., Tokushige N. A word problem in Artin semigroups // Discrete Math. 1992. V. 104. P. 159-166.

[10] Servatius H. An Artin relation of length $n$ in the braid group // Discrete Math. 1992. V. 104. P. 185-189. 